\title{
Logistics Information Network Design and Implementation
}

\author{
Yin Hang \\ Eegineeing Training Center \\ Shengyang Aerospace University \\ Shengyang China
}

\begin{abstract}
With computer technology, network technology and information technology, rapid development, to bring great modern work of the change in the life of modern man also given a lot of convenience. Logistics and transport industry today is moving in the pursuit of efficient, low-cost, reliable direction. Wide rise of the logistics information network, while reducing material consumption, on the other hand increased labor productivity. Information technology, automation, networking, and has become a distinct characteristic of modern logistics. Only by relying on modern logistics enterprise of scientific logistics and logistics information management, will be storage, transportation, distribution, messaging and other functions into one. To provide customers with safe, high quality, fast, accurate and modern logistics services. Therefore, the establishment of a sound and effective logistics information management system is necessary.System using JSP + MS SQL. SERVER with B / S model development, a safe, convenient, easy configuration and so on. This review describes the system requirements analysis based on the proposed system design objectives, system structure and function of the overall module design. Logistics management system from a system requirements analysis, design, and detailed design to achieve specific coding, functional testing strictly followed the ideas of software engineering. This system mainly realizes the information of all kinds of information release, check, information modification, information delete, auxiliary tools, member center and background management function, can be easily in logistics information timely, effective management.
\end{abstract}

Keywords-SQL; JSP; logistics management systems; Software Engineering

\section{INTRODUCTION}

With the information's era coming, the level of science and technology is continuously improved, especially the powerful function of increasingly mature computer science has been deeply realized by the human. The computer science is entering into every field of human society, playing an more and more important role and far-reaching impact on human being.

In today's society, there's an increasingly competition in all the industries, among them has the logistics transportation industry. The industry development requires the logistics practitioners to use more effective information means to expand its own business space, reduce operation cost, improve management and decision-making efficiency. Too much manual operation and some unscientific management method of traditional logistics management cannot meet comprehensive information management of the enterprise, which is bound to affect practitioner's working efficiency, decrease market competitive capacity. A successful logistics practitioner should regard the enterprise economic profit as the final goal of operation, the core of management is how to improve enterprise economic profit.

\section{DEVELOPING BACKGROUND}

\section{A. The Market Demand for Logistics Management System}

Since reform and opening, China's economy and world's economy is more and more closely connected. There have more than 400 enterprises in Fortune Global 500 have entered in China market. The entry of foreignfunded enterprise, on the one hand, it brings modern logistics concept and advanced operating model, on the other hand, it also promotes the improvement of modern logistics service level in China's enterprise. With the rapid development of the world economy, global digitalization, networking and information has become the main features of market economy era.

\section{B. Research Status at Home and Abroad}

For current development of logistics information system, which has entered into a mature stage,and turn to the research of complex functions. Although started late in China,it still has a rapid development in recent years. Most of the domestic enterprise logistics information system is geared to the basic flow control, such as inventory controlling system,cargo tracking and query system etc. And it uses traditional customer/server mode(C/S) structure. However, as rapid development of IT network technology, there's an increasingly demand for information, domestic enterprise starts to research the development of the browser/server (B/S)mode.Under this structure, system working interface is implemented through IE browser, which brings great convenience for cargo information updating and effective management.

\section{THEORETICAL BASIS}

\section{A. JSP Technology}

JSP has become the most popular network programming language, which is being widely used in ecommerce, e-government and software industry. JSP is a dynamic web technology, it inherits the advantages of Java language, realizes independence from the platform.JSP has the advantages like cross-platform, high efficiency and 
easy to operate etc. As long as with basic knowledge about programming design, it will be quite easier to learn JSP.

\section{1) Separation of Content and Presentation}

With JSP technology, Web page developer can use HTML or XML symbol to design and formatting the final page. By using JSP symbol or small script go generate dynamic content on the page. The generated content logic is encapsulated in the symbol and JavaBean component, and bundled in small script, all the scripts are executed on the server.

2) Platform

ASP only can operate under Windows platform, while owning to its strong Java language, JSP can operate under almost all the operation system platform. With a plug-in JRUN, IIS of NT also can support JSP, the famous Web server Apache has now been able to support JSP, which is widely operated in NT, UNXI, LINUX.

3) Reusability

It can be encapsulated in JavaBeans component, through which to implement some complicated processing, and present the processing outcome by JSP calls. On the one hand it makes developers can concentrate on component developing; on the other hand, developer who written JSP can use the programme in several places instead of caring about details realization.

4) Database Connection Technology

Java programme is connected with database through JDBC drive programme.Quite number of database has JDBC drive programme,JDBC-ODBC pattern provide a bridge for JDBC and ODBC drive connection.

\section{B. Database Technology}

Database technology derived from the late 1960s and early 70s. It's main purpose is to effectively manage and store a large amount of data resources. Database technology primarily researches how to store, use and manage the data. In recent years, the development of database technology and computer networking technology mutually penetrates and promotes, which has become two widely used fields with rapid development.

\section{B/S MODE}

$\mathrm{B} / \mathrm{S}$ ( Browser/Server)mode also known as B/S structure.It's the $\mathrm{C} / \mathrm{S}$ mode expansion with the rising of Internet technology.B/S mainly uses a maturing WWW browser technology, combining multilingual Script language(VBScript, JavaScript etc.) and ActiveX technology, which is a newly software system structure technology.

In B/S system structure, users make request to several servers distributed on the network through browser,servers process the request from the browser and return the information required by the users to the browser. The left like data request, processing, outcome return and dynamic webpage generation, visiting to database and execution of application programme will be finished by Web Server.Along with Windows embeds the browser technology inside of operation system, this kind of structure has become the first choice architecture of today's application software. Obviously, compared to traditional $\mathrm{C} / \mathrm{S}$ structure application programme, $\mathrm{B} / \mathrm{S}$ programme is a very great progress.

\section{SYSTEM REQUIREMENT ANALYSIS}

\section{A. The Current Business System Description}

The enterprise uses traditional artificial management in current logistics management. It requires a lot of manpower and material resources for goods registration, renewal,transfer and documents acceptance. The whole good logistics information management of each link All the relies on artificial participation to finish all the tasks.

\section{B. Main Problems Analysis in Current System}

In modern society, logistics management information system is a widely demanded system in IT company and ecommerce website.however,people have been used traditional artificial management. This kind of management have lots of deficiencies, such as low efficiency,more troublesome, in addition, with the increasing of logistics management project, the workload also arises. This will inevitably increase the workload and labor intensity of logistics managers, and will bring lots of difficulties for logistic management information searching, updating and maintenance.

\section{Feasibility Analysis}

Logistics information network provide the logistics enterprises with dynamic information, supply and demand information browsing and query functions, help the enterprises to learn more about the industry dynamic information, improve enterprise operation efficiency. The powerful function of logistics information network becomes more and more important for logistics industry stakeholders. Through the website the enterprise can adjust the development tendency more timely so as to adapt to market changes.

\section{OVERALL ANALYSIS OF THE SYSTEM}

\section{A. System Module Structure}

Back end system function structure of the logistics information network.

Back end system function structure of logistics information network.

\section{B. Objective of System Designed}

The overall objective of the system is to analyze and design as an important part in integrated planning of logistics management system, making full use of computer network technology, transmission technology and database technology ect.to realize a system with remote maintenance,storage space management and all kinds of inventory dynamic query. It can ensure the information flow, reduce intermediate links and optimize the management process. Through centralized management and multilevel authorization safety system to ensure the normal operation of the system, provide accurate and reliable statistics at any time; meanwhile, to strive to give consideration of system universality, generalization, steady and transportability.Specific analysis and design process should follow the basic principle below:

Reliability: Lay emphasize on using mature and practical technology, make the highest input-output ratio 
of system construction, ensure a long-term secure operation of the system.

Security: The system should have necessary safety and security measures.

Fault tolerance: The system should have a specific fault tolerant capacity. Should have the ability of tips or automatically elimination for customers mis-operation.

Adaptability: The system should be constantly developed and improved so as to has a widely adaptability for all the algorithm and system.

\section{E-R Diagram Design}

ER diagram of logistics information network system,.

\section{SYSTEM FUNCTION MODULE}

\section{A. Summary of System Module}

Logistics information network is a professional logistics information website, which is composed of front end information browsing, management for released information from members and back end management.

Frond end mainly includes the function modules like the release of all kinds of information, information query, modify, delete, auxiliary means, member center and back end $\log$ in etc.

- Site navigation: primary includes banner advertising and main function navigation two parts.

- Information release module: includes enterprise information release, vehicle and goods information release these three parts.

- Information view module: includes enterprise information, vehicle, goods, logistics and notice information view these 5 parts.

- Information modify module: includes enterprise information, vehicle and goods information modify.

- Information delete module: includes enterprise information, vehicle information and goods information delete.

- Information query module: to realize classified information query.

- Auxiliary means: national highway information and license plate number information query.

- Member center:mainly includes member log in, registration and password retrieve.

- Enterprise business links: used for displaying links information.

- Back end log in entry: to provide an entry for administrator to enter the back end.

Back end management module

Back end mainly includes enterprise information, notice information, vehicle information, logistics knowledge, goods information and member management.

\section{SYSTEM TESTING}

English is System Testing, which combines the other elements like the confirmed software, computer's hardware and peripheral and network to take kinds of assembly and validation test of information system. System testing aims at the testing for the complete product system, the purpose of it is to verify if the system meets the definition of demand specification, and find out unconformity or contradiction with the demand specification, so that to put forward a more perfect solution. If there's problems after testing, should find out the reason for error and position through debugging, then make corrections. For the black-box testing based on the whole demand specification, all the joint components should be covered. Besides the software required to be tested, the subject also includes the hardware, peripheral even some data, supportive software and port etc.

Order Testing Items Input Instructions(operation) Output instructions(expected outcome)

- Login module-login function Present main interface after the user input correct user name and password, and successfully log in.

- Login module-modify the password The password is modified correctly after the user

log in successfully, input old password and new password.

- Login module-Create new user Input new user name and password, create a new user successfully.

- Login module-log in failed. Input incorrect user and password, log in failed, automatically log out.

- Login module-default user. There's default user in database, use default user name and password, log in successfully.

Order Testing Items Input Instructions(operation) Output Instructions(expected outcome)

- System management module-delete function. Click delete return to the front page after deleting.

- System management module-edit function.Click edit password is modified successfully.

Order Testing Items Input Instructions(operation) Output Instructions(expected outcome)

- System management module-delete function Click delete return to front page of system after deleting.

- System management module-edit function Click edit the password is modified successfully.

After each module is designed, it requires to test its function one by one to check if it reaches expected effect.After finishing testing of each module, overall testing is also required, once finish this testing, the whole system is preliminary completed.

Problems in the test:

Chinese character is previewed as error codes

In JSP page, the position of Chinese character often has messy code appeared, here's a solution:

Configurate the following information in web.xml.

$<$ filter>

$<$ filter-name $>$ filter $</$ filter-name $>$ $<$ filter-

class >com.niit.struts.filter.CharSetFilter $</$ filter-class $>$ $<$ init-param>

$<$ param-name $>$ charset $</$ param-

name> $<$ param-value $>$ UTF-8</param-

value>

$</$ filter $>$
<filter-mapping $>$
$\quad$ <filter-name $>$ filter $</$ filter-name $>$




\section{url-pattern $>/ *</$ url-pattern $>$}

Add a new classification and take configuration in src content.the following is core code:

public class CharSetFilter implements Filter \{

String charSet = "";

public void init(FilterConfig config) throws ServletException \{

charSet

config.getInitParameter("charset");

\}

public void doFilter(ServletRequest request,

ServletResponse response,

IOException, ServletException \{

FilterChain chain) throws

(HttpServletRequest) request;

HttpServletRequest httpRequest =

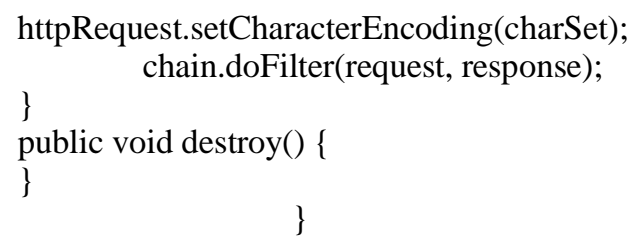

\section{CONCLUSTION}

As a new dynamic webpage design technology, JSP has it own uniqueness, with convenient access and easier language grammar, which reduces the burden of developers.
This writing make me realize the importance of design in software development process. To design a good software structure(including related module integration and call relation, related module independence consideration, database structure design and actual software process) plays a key role for final software quality and middle development task.

Due to a preliminary study for JSP technology, and without adequate experiences on developing big project, which make a large code repetitive; however, at the same time, I found the studying pleasure in website design and code writing. I plan to further improve the design in future, and rewrite the whole website code and increase website functions. If condition permits, the portability of Java also can be played to research the relevant cross-platform portability.

\section{REFERENCES}

[1] Qinghong computer office, JSP Programming Skill,China Machine Press. May, 2004

[2] Zhu hong, Si Guangya, JSP web Programming Instruction,Electronic Industry Press. Sep.,2001

[3] Sai Kuichun, JSP Engineering Application and Project Practice,China Machine Press,August. 2002

[4] (US)Hall writes, Zhong Ming etc. translate, Authoritative Guidance of Servlet and JSP, China Machine Press. 2002-10-1

[5] Liu Bin Ed., Advanced Course of JSP Database Tsinghua University Press. 2006-3-1

[6] Feisi Science and Technology Product Research Center compile, Advanced Management and Development of SQLServer2000, Electronic Industry Press. 2002-1-1

[7] Zhao Jie etc. compile, Database Principle and Application(SQLServer),Post and Telecom Press. 2006-7-1

[8] Matt Welsh. Tigris: A Java-based Cluster I/O System.Co iitp nt er Science Division University of California. 2007 